\title{
On Five-diagonal Splitting for Cubic Spline Wavelets with Six Vanishing Moments on a Segment
}

\author{
BORIS SHUMILOV \\ Department of Applied Mathematics \\ Tomsk State University of Architecture and Building \\ 2 Solyanaya, Tomsk, 634003 \\ RUSSIA \\ sbm@tsuab.ru
}

\begin{abstract}
In this study, we use the zeroing property of the first six moments for constructing a splitting algorithm for cubic spline wavelets. First, we construct a new system of cubic basic spline-wavelets, realizing orthogonal conditions to all polynomials up to fifth degree. Then, using the homogeneous Dirichlet boundary conditions, we adapt spaces to the closed interval. The originality of the study consists of obtaining implicit relations connecting the coefficients of the spline decomposition at the initial scale with the spline coefficients and wavelet coefficients at the nested scale by a tape system of linear algebraic equations with a non-degenerate matrix. After excluding the even rows of the system, in contrast to the case with two zero moments, the resulting transformation matrix has five (instead of three) diagonals. The problems of modeling automobile road pavements using laser scanned data are described. The results of numerical experiments on visualizing the designed road from the processed laser points are also presented.
\end{abstract}

Key-Words: - $B$-splines, wavelets, implicit decomposition relations, laser scanning, data processing, roads modeling

Received: February 3, 2020. Revised: July 25, 2020. Accepted: August 26, 2020. Published: November 3, 2020.

\section{Introduction}

Increasing the efficiency of digital information processing is an urgent task of modern applied mathematics. This is especially noticeable in the areas related to computer graphics. The problem of threedimensional modeling of open areas of the terrain, extended objects of transport, industrial and civil construction is now becoming more and more urgent. The requirements for a realism of generated images are constantly growing, which ultimately leads to an increase in computational costs. At the same time, for many applications (for example, visualization of laser scanning data of automobile roads), very high processing speed is required. One of the ways to increase the efficiency of digital information processing is the use of methods based on the multiscale representation of graphical objects. A multiscale representation is a multilayer structure, the first layer of which contains sufficient information to coarse (low resolution) approximation of an object; when adding information from each subsequent layer, the level of details gradually increases until the object is fully restored (that is, with maximum resolution). With the methods based on multiscale representation, a wide range of problems of processing numerical information can be solved, including noise elimination, visualization of graphical objects, reduction of data vol- umes by removing redundant and irrelevant information, thereby reducing the computational cost of subsequent processing, etc. Among these methods, algorithms for processing multiscale representations based on wavelet analysis, which are quite simple and effective in implementation, have recently become widespread. This transformation provides a nonstationary time-frequency representation [1], which attracts much attention from researchers in areas such as image compression, image noise processing, signal processing, computer graphics, and pattern recognition.

Wavelets are short or quickly damped wave functions (bursts), a set of shifts and compressions of which together generate the space of measurable functions for the whole numeric axis [2-5]. If there are several such waves, then multiwavelets [6-9] appear. Multiwavelets became the subject of special attention after it was shown that symmetry and orthogonality can be simultaneously achieved, which is impossible $[4,6]$ in the case of a traditional scalar wavelet. Because compressions the wavelets reveal varying scales of detail differences between the characteristics of the measured signal, and due to shifts, they can analyze the properties of a signal at different points throughout the studied interval. In the analysis of non-stationary signals, the locality prop- 
erty of wavelets provides them an advantage over the Fourier transform, which gives only global information about properties of a signal under study; since the basic functions used in this case (sine and cosine) have infinite support. In solving problems of numerical analysis, since wavelets transform a system of basic functions with distributed parameters into a system with lumped parameters, such a basis is more efficient in terms of stability and convergence [10]. The unique properties of wavelets allow designing a basis in which the data representation can be expressed with a small number of non-zero coefficients. This property makes wavelets attractive for data compression, including video and audio information. Wavelet transform can be viewed as one of the methods of primary signal processing to improve the efficiency of its compression. In this case, direct compression is performed by classical methods after its preprocessing only for significant coefficients of the wavelet decomposition of the signal, and its reconstruction according to these coefficients is performed at the stage of restoration (decompression).

In the work of the author [11], non-orthogonal wavelets of the third degree with the first two zero moments, that is, orthogonal to polynomials of the first degree, were considered, the existence of finite implicit decomposition relations was proved, and an effective algorithm for wavelet analysis based on them was justified. This article attempts to find out if there is an even-odd splitting algorithm for nonorthogonal cubic wavelets with the first six zero moments; the lack of a strict diagonal dominance in the splitting system is discussed; the results of numerical experiments on roads laser data processing are presented.

\section{Construction of cubic spline wavelets with six vanishing moments on an interval}

Let $V_{L}$ denotes a space of cubic splines of smoothness $C^{2}$ on a segment $[a, b]$ with a uniform grid consisting of the nodes $\Delta^{L}: x_{i}=a+h \cdot i, i=0,1, \ldots, 2^{L}$, $h=(b-a) / 2^{L}$, and the basic functions $\varphi_{3}(v-i) \forall i$, where $v=(x-a) / h$, with the centers in integers, are generated by means of compressions and displacements of the function of the form [12, p. 89]:

$$
\varphi_{3}(t)=\frac{1}{6} \sum_{j=0}^{4}\left(\begin{array}{l}
4 \\
j
\end{array}\right)(-1)^{j}(t-j)_{+}^{3},
$$

where $t_{+}^{n}=(\max \{t, 0\})^{n}$.

Then these functions satisfy the calibration rela- tion $[5, \mathrm{p} .91]$ :

$$
\varphi_{3}(t)=\frac{1}{8} \sum_{k=0}^{4}\left(\begin{array}{l}
4 \\
k
\end{array}\right) \varphi_{3}(2 t-k) .
$$

We use here for constructing a basis for $W_{L-1}$ the cubic wavelet orthogonal to all polynomials of the fifth degree of the following form [13]

$$
w_{3}(t)=\frac{1}{8} \sum_{k=0}^{6}(-1)^{k}\left(\begin{array}{l}
6 \\
k
\end{array}\right) \varphi_{3}(2 t-k) .
$$

It has six zero moments

$$
\int_{-\infty}^{\infty} x^{k} w_{3}(x) d x=0, k=0,1, \ldots, 5,
$$

in addition, these functions have the following supports:

$$
\operatorname{supp} \varphi_{3}=[0,4], \operatorname{supp} w_{3}=[0,6] .
$$

Unfortunately, to construct the bases of the spline and wavelet spaces on a finite segment $[a, b]$, the functions $\varphi_{3}(v-i)$ and $w_{3}(v-i)$ cannot simply be truncated when going beyond the ends of the segment. There are two approaches to solving this problem: the first is the introduction of additional nodes to the right and the left of the ends of the segment and the other is the use of multiple nodes at points $a, b$. In the theory of wavelets, the second approach is more popular. Additionally, we have to keep the symmetry of an expanded function on a finite interval. This requires arranging the bases so that the location of basic functions can be symmetrical concerning the center of the interval. To accomplish this arrangement and having in mind the desired property of splitting algorithms, we will locate the centers of supports of $w_{3}(v-i)$ at odd nodes $j=5,7, \ldots, 2^{L}-5$, to receive in the result $2^{L-1}-4$ degrees of freedom. To discuss the number of boundary wavelets, let us calculate firstly the quantity of the basic splines $\varphi_{3}(v-i), i=0,1, \ldots, 2^{L}-4$, fully nested in the interval $[a, b]$, to receive in the result $2^{L}-3$ degrees of freedom. If the grid $\Delta^{L-1}$ is obtained from $\Delta^{L}$ by deleting every second node, then the corresponding basic functions $\varphi_{3}(v / 2-i) \forall i$, whose supports are twice as wide and centers at even nodes of the grid $\Delta^{l}$, provide $2^{L-1}-3$ degrees of freedom. Since the difference of the degrees of freedoms in both scales has to be equal to the number of added wavelets, four boundary wavelets and four boundary basic splines are necessary to obtain the nested resolution approximation. Recall, that the whole dimension of $V_{L}$ equals $2^{L}+3$. So to make true the nested resolution argument we ought to neglect two degrees of freedoms in each scale of spline approximation. To do so we simply impose the following additional conditions on functions being approximated: 
$f(a)=f(b)=0$. This assumption facilitates the construction of wavelets near the ends of a finite interval and the condition of the complement of dimensions of the considered spaces is satisfied, i.e.

$$
\operatorname{Dim}\left(V_{L}\right)=2^{L}+1=\operatorname{Dim}\left(V_{L-1}\right)+\operatorname{Dim}\left(W_{L-1}\right) .
$$

Then the corresponding left basic functions have the form [14]

$$
\begin{array}{r}
\varphi_{b 1}(t)=\frac{7}{4} t_{+}^{3}-\frac{9}{2} t_{+}^{2}+3 t_{+}^{1}-2(t-1)_{+}^{3}, 0 \leq t \leq 2, \\
\varphi_{b 2}(t)=\frac{3}{2} t_{+}^{2}-\frac{11}{12} t_{+}^{3}+\frac{3}{2}(t-1)_{+}^{3}-\frac{3}{4}(t-2)_{+}^{3}, \\
0 \leq t \leq 3,
\end{array}
$$

and satisfy the calibration relations

$$
\begin{aligned}
\varphi_{b 1}(t)= & \frac{1}{2} \varphi_{b 1}(2 t)+\frac{3}{4} \varphi_{b 2}(2 t)+\frac{3}{16} \varphi_{3}(2 t),(3) \\
\varphi_{b 2}(t)= & \frac{1}{4} \varphi_{b 2}(2 t)+\frac{11}{16} \varphi_{3}(2 t)+ \\
& +\frac{1}{2} \varphi_{3}(2 t-1)+\frac{1}{8} \varphi_{3}(2 t-2)
\end{aligned}
$$

As to boundary basic wavelets to illustrate the main idea of the studying we shall use here the cubic wavelets that are orthogonal to all third-degree polynomials [14],

$$
\begin{array}{r}
w_{b 1}(t)=6 \varphi_{b 1}(2 t)-\frac{57}{5} \varphi_{b 2}(2 t)+\frac{919}{100} \varphi_{3}(2 t)- \\
-\frac{116}{25} \varphi_{3}(2 t-1)+\varphi_{3}(2 t-2), \\
w_{b 2}(t)=\frac{7}{3} \varphi_{b 2}(2 t)-\frac{319}{60} \varphi_{3}(2 t)+\varphi_{3}(2 t-3)+ \\
+\frac{101}{15} \varphi_{3}(2 t-1)-\frac{25}{6} \varphi_{3}(2 t-2) .
\end{array}
$$

They have the following supports

$$
\operatorname{supp} w_{b 1}=[0,3], \operatorname{supp} w_{b 2}=[0,3.5],
$$

and, accordingly, they have four zero moments

$$
\int_{0}^{3} x^{k} w_{b 1}(x) d x=\int_{0}^{3.5} x^{k} w_{b 2}(x) d x=0
$$

for $k=0,1,2,3$.

At the right end of the segment, the basic functions mirror the functions $\varphi_{b 1,2}(t), w_{b 1,2}(t)$. As a result, for any grid $\Delta^{L}, L \geq 2$, a third-degree spline with zero boundary conditions can be represented as

$$
\begin{array}{r}
S^{L}(v)=C_{-2}^{L} \varphi_{b 1}(v)+C_{-1}^{L} \varphi_{b 2}(v)+ \\
+\sum_{i=0}^{2^{L}-4} C_{i}^{L} \varphi_{3}(v-i)+C_{2^{L}-3}^{L} \varphi_{b 2}\left(2^{L}-v\right)+ \\
+C_{2^{L}-2}^{L} \varphi_{b 1}\left(2^{L}-v\right), 0 \leq v \leq 2^{L},
\end{array}
$$

where the coefficients $C_{i}^{L} \forall i$ are the solution, for example, of the interpolation problem:

$$
\begin{aligned}
S^{L}(i) & =f\left(x_{i}\right), i=1,2, \ldots, 2^{L}-1, \\
\left(S^{L}\right)^{\prime}(i) & =h \cdot f^{\prime}\left(x_{i}\right), i=1,2^{L}-1 .
\end{aligned}
$$

\section{Construction of the defining system of wavelet transform equations}

For further reasoning, it is convenient to write the basic spline functions in the form of a single row matrix,

$$
\varphi^{L}(\cdot)=\left[\varphi_{b 1}(\cdot), \varphi_{b 2}(\cdot), \varphi_{3}(\cdot), \varphi_{3}(\cdot-1), \ldots\right.
$$

$$
\left.\ldots, \varphi_{3}\left(\cdot-2^{L}+4\right), \varphi_{b 2}\left(2^{L}-\cdot\right), \varphi_{b 1}\left(2^{L}-\cdot\right)\right]
$$

and to arrange the spline coefficients in the form of a vector,

$$
C^{L}=\left[C_{-2}^{L}, C_{-1}^{L}, C_{0}^{L}, \ldots, C_{2^{L}-3}^{L}, C_{2^{L}-2}^{L}\right]^{T} .
$$

Then the formula (7) is rewritten as

$$
S^{L}(\cdot)=\varphi^{L}(\cdot) C^{L} .
$$

Similarly, for $L \geq 4$ we can write the basic wavelet functions in the form of a row matrix as

$$
\begin{aligned}
& \psi^{L-1}(\cdot)=\left[w_{b 1}(\cdot), w_{b 2}(\cdot), w_{3}(\cdot), w_{3}(\cdot-2), \ldots\right. \\
& \left.\ldots, w_{3}\left(\cdot-2^{L}+5\right), w_{b 2}\left(2^{L}-\cdot\right), w_{b 1}\left(2^{L}-\cdot\right)\right] .
\end{aligned}
$$

The corresponding coefficients of the decomposition wavelets at the scale $L-1$ are assembled into a vector,

$$
D^{L-1}=\left[D_{-1}, D_{0}, \ldots, D_{2^{L-1}-2}\right]^{T} .
$$

Then, for a scale of the expansion of $L-1$, the functions $\varphi^{L-1}(\cdot)$ and $\psi^{L-1}(\cdot)$ can be written as linear combinations of the functions $\varphi^{L}(\cdot)$ :

$$
\varphi^{L-1}(\cdot)=\varphi^{L}(\cdot) P^{L} \text { and } \psi^{L-1}(\cdot)=\varphi^{L}(\cdot) Q^{L},
$$

where the columns of the matrix $P^{L}$ are composed of the relation coefficients (1) and (3), (4) since each wide basic function within the approximation interval can be constructed from five narrow basic functions, each wide basic function at the ends of the interval can be constructed from three or four narrow basic functions while the elements of the columns of the matrix $Q^{L}$ are composed of the relation coefficients (2) and (5), (6).

Consequently, the following equalities hold:

$$
\begin{aligned}
\varphi^{L}(\cdot) C^{L} & =\varphi^{L-1}(\cdot) C^{L-1}+\psi^{L-1}(\cdot) D^{L-1}= \\
& =\varphi^{L}(\cdot) P^{L} C^{L-1}+\varphi^{L}(\cdot) Q^{L} D^{L-1} .
\end{aligned}
$$


Thus, the process of obtaining $C^{L}$ from $C^{L-1}$ and $D^{L-1}$ can be written in the form

$$
C^{L}=P^{L} C^{L-1}+Q^{L} D^{L-1}
$$

or, using the notation for block matrices,

$$
C^{L}=\left[P^{L} \mid Q^{L}\right]\left[\frac{C^{L-1}}{D^{L-1}}\right] .
$$

Define the block matrix that inverse to the matrix $\left[P^{L} \mid Q^{L}\right]$ :

$$
\left[\frac{A^{L}}{B^{L}}\right]=\left[P^{L} \mid Q^{L}\right]^{-1} .
$$

Then the process of creating a version with a coarse resolution, $C^{L-1}$, characterized by fewer coefficients, can be expressed by the matrix equality

$$
C^{L-1}=A^{L} C^{L},
$$

where $A^{L}$ is a matrix of dimension $\left(2^{L-1}+1\right) \times$ $\left(2^{L}-1\right)$.

In this case, the lost parts are collected in another vector $D^{L-1}$, defined by the expression

$$
D^{L-1}=B^{L} C^{L},
$$

where $B^{L}$ is a matrix of dimension $2^{L-1} \times\left(2^{L}-1\right)$. Matrices $A^{L}$ and $B^{L}$ are called analysis filters, and matrices $P^{L}$ and $Q^{L}$ are called synthesis filters [4, p. 95], [10, p. 198].

Unfortunately, as it is easy to see, the matrices that inverse to $\left[P^{L} \mid Q^{L}\right]$ lose their sparse structure. The essence of the approach proposed in [4, p. 96] for such cases is that $C^{L-1}$ and $D^{L-1}$ can be calculated from $C^{L}$ by solving the system of linear equations (9). Moreover, they proposed to remake the matrix $\left[P^{L} \mid Q^{L}\right]$ into a tape matrix by simply changing the order of the unknowns so that the columns of the matrices $P^{L}$ and $Q^{L}$ were interleaved. Thus, the operation of wavelet decomposition can be performed without explicit representation and the use of a filter block. Nevertheless, although the solvability of the resulting system is guaranteed by the linear independence of the basic functions, it has seven diagonals, which are quite large. Moreover, the question of its good conditioning remains open. As can be easy seen, the resulting system of equations does not have diagonal dominance, which can make it difficult to analyze

\begin{tabular}{|c|c|c|c|c|c|c|c|c|c|c|c|c|c|}
\hline 904 & 600 & 15920 & 340 & $\frac{123680}{87}$ & 0 & 0 & 0 & & & & & & \\
\hline 0 & -908 & 0 & 0 & 0 & 0 & 0 & 0 & & & & & & \\
\hline 1338 & 1557 & 9111 & 301 & $\frac{25654}{29}$ & 5 & 5 & 0 & & & & & & \\
\hline 0 & 0 & 0 & -116 & 0 & 0 & 0 & 0 & & $\vdots$ & $\vdots$ & $\vdots$ & $\vdots$ & $\vdots$ \\
\hline 162 & 216 & -4626 & 25 & $-\frac{3969}{29}$ & 51 & 60 & 5 & & 0 & 0 & 0 & 0 & 0 \\
\hline 0 & 0 & 0 & 0 & 0 & -16 & 0 & 0 & $\ddots$ & 15 & 0 & 0 & 0 & 0 \\
\hline 0 & 0 & -261 & 0 & 144 & 51 & 126 & 51 & $\ddots$ & 0 & 0 & 0 & 0 & 0 \\
\hline 0 & 0 & 0 & 0 & 0 & 0 & 0 & -16 & $\ddots$ & 144 & 0 & -261 & 0 & 0 \\
\hline 0 & 0 & 0 & 0 & 15 & 5 & 60 & 51 & $\ddots$ & 0 & 0 & 0 & 0 & 0 \\
\hline 0 & 0 & 0 & 0 & 0 & 0 & 0 & 0 & $\ddots$ & $-\frac{3969}{29}$ & 25 & -462 & 216 & 162 \\
\hline \multirow[t]{3}{*}{$\vdots$} & $\vdots$ & $\vdots$ & $\vdots$ & $\vdots$ & $\vdots$ & 5 & 5 & $\ddots$ & 0 & -116 & 0 & 0 & 0 \\
\hline & & & & & & 0 & 0 & $\ddots$ & $\frac{25654}{29}$ & 301 & 9111 & 1557 & 1338 \\
\hline & & & & & & $\vdots$ & $\vdots$ & $\ddots$ & $\begin{array}{c}0 \\
123680 \\
87\end{array}$ & $\begin{array}{c}0 \\
340\end{array}$ & $\begin{array}{c}0 \\
15920\end{array}$ & $\begin{array}{c}-908 \\
600\end{array}$ & $\begin{array}{c}0 \\
904\end{array}$ \\
\hline
\end{tabular}
large-size wavelet data.

\section{Splitting algorithm}

Let for resolution scales $L \geq 4$, the matrix $G^{L}$ of size $\left(2^{L}+1\right) \times\left(2^{L}+1\right)$ has the form
Here diagonal points mean that the preceding two columns are repeated the appropriate number of times, each time moving two positions right and down, while the matrix $R^{L}$ is compound from two blocks according to $2^{L-1}+1$ basic spline functions of $V_{L-1}$ and $2^{L-1}$ basic wavelets of $W_{L-1}$ : 


\begin{tabular}{|c|c|c|c|c|c|c|c|c|c|c|c|c|}
\hline 908 & 0 & 16360 & 380 & $\frac{128200}{87}$ & 0 & 0 & 0 & & & & & \\
\hline 696 & 928 & 0 & 0 & 0 & 0 & 0 & 0 & & & & & \\
\hline 0 & 0 & -2088 & 0 & 0 & 24 & 24 & 0 & $\vdots$ & $\vdots$ & $\vdots$ & $\vdots$ & $\vdots$ \\
\hline 0 & 0 & 0 & 0 & 72 & 24 & 80 & 24 & 0 & 0 & 0 & 0 & 0 \\
\hline 0 & 0 & 0 & 0 & 0 & 0 & 24 & 24 & 72 & 0 & 0 & 0 & 0 \\
\hline \multirow[t]{2}{*}{$\vdots$} & $\vdots$ & $\vdots$ & $\vdots$ & $\vdots$ & $\vdots$ & 0 & 0 & 0 & 0 & -2088 & 0 & 0 \\
\hline & & & & & & $\vdots$ & $\vdots$ & $\begin{array}{c}0 \\
128200 \\
87\end{array}$ & $\begin{array}{c}0 \\
380\end{array}$ & $\begin{array}{c}0 \\
16360\end{array}$ & $\begin{array}{c}928 \\
0\end{array}$ & $\begin{array}{l}696 \\
908\end{array}$ \\
\hline 75 & 100 & 1290 & 25 & $\frac{3310}{29}$ & 0 & 0 & 0 & & & & & \\
\hline 0 & 0 & 1044 & 0 & 84 & 0 & 0 & 0 & & & & & \\
\hline 0 & 0 & 0 & 0 & -48 & -16 & -16 & 0 & $\vdots$ & $\vdots$ & $\vdots$ & $\vdots$ & $\vdots$ \\
\hline 0 & 0 & 0 & 0 & 0 & 0 & -16 & -16 & 0 & 0 & 0 & 0 & 0 \\
\hline \multirow[t]{2}{*}{$\vdots$} & $\vdots$ & $\vdots$ & $\vdots$ & $\vdots$ & $\vdots$ & 0 & 0 & -48 & 0 & 0 & 0 & 0 \\
\hline & & & & & & $\vdots$ & $\vdots$ & $\begin{array}{c}84 \\
\frac{3310}{29}\end{array}$ & $\begin{array}{c}0 \\
25\end{array}$ & $\begin{array}{l}1044 \\
1290\end{array}$ & $\begin{array}{c}0 \\
100\end{array}$ & $\begin{array}{c}0 \\
75\end{array}$ \\
\hline
\end{tabular}

Here diagonal points mean that the preceding two columns are repeated the corresponding number of times while going right two positions, each time moving one position down. The last five columns of the matrices $G^{L}$ and two blocks of $R^{L}$ mirror the first five columns; the empty positions of matrices are equal to zero.

We propose to perform calculations based on our previously developed procedure $[15,16]$ of the evenodd splitting of wavelet decomposition of the form (8), connecting the basic functions of the space of splines on a dense grid, the basic functions on a sparse grid, and wavelets by finite implicit relations of the expansion with three non-empty diagonals and strict diagonal dominance. For the type of wavelets presented above, similar results are valid, which, using the notations above, can be represented in the following form.

Theorem 1 For any scale of resolution $L \geq 4$, the matrix of the wavelet decomposition of cubic splines satisfies the equality

$$
\left[P^{L} \mid Q^{L}\right] R^{L}=G^{L}
$$

From the notation of the matrix equation (10) it can be seen that for each column of the matrix $G_{j}^{L}$ the corresponding column of the matrix $R_{j}^{L}$ is nothing more than a solution of a system of linear equations of the form

$$
\left[P^{L} \mid Q^{L}\right] R_{j}^{L}=G_{j}^{L}
$$

Now it is seen that for the elements of the columns of the matrix $R^{L}$, the system of equations (11) has only five mirrored solutions for the right and left ends of the segment $[a, b]$ and two solutions in the variable part of the system, depending on the number of internal nodes of the grid $\Delta^{L}$. All other solutions differ only in the placement of these elements in columns. Since each row of the matrix, $\left[P^{L} \mid Q^{L}\right]$ contains only a few nonzero elements, these systems themselves have a small dimension. For each variant, the resulting systems turn out to be consistent, but their ranks are less than the number of unknowns, so each system provides some freedom in choosing the most compact solution in the sense of the least number of nonzero values and regular solution in the sense of zeroing out the even right-hand sides. Therefore, it is not difficult to obtain these solutions or at least check the available solutions using manual counting. Let's find their explicit representation.

For the matrix $\left[P^{L} \mid Q^{L}\right]$ the seven-diagonal matrix 


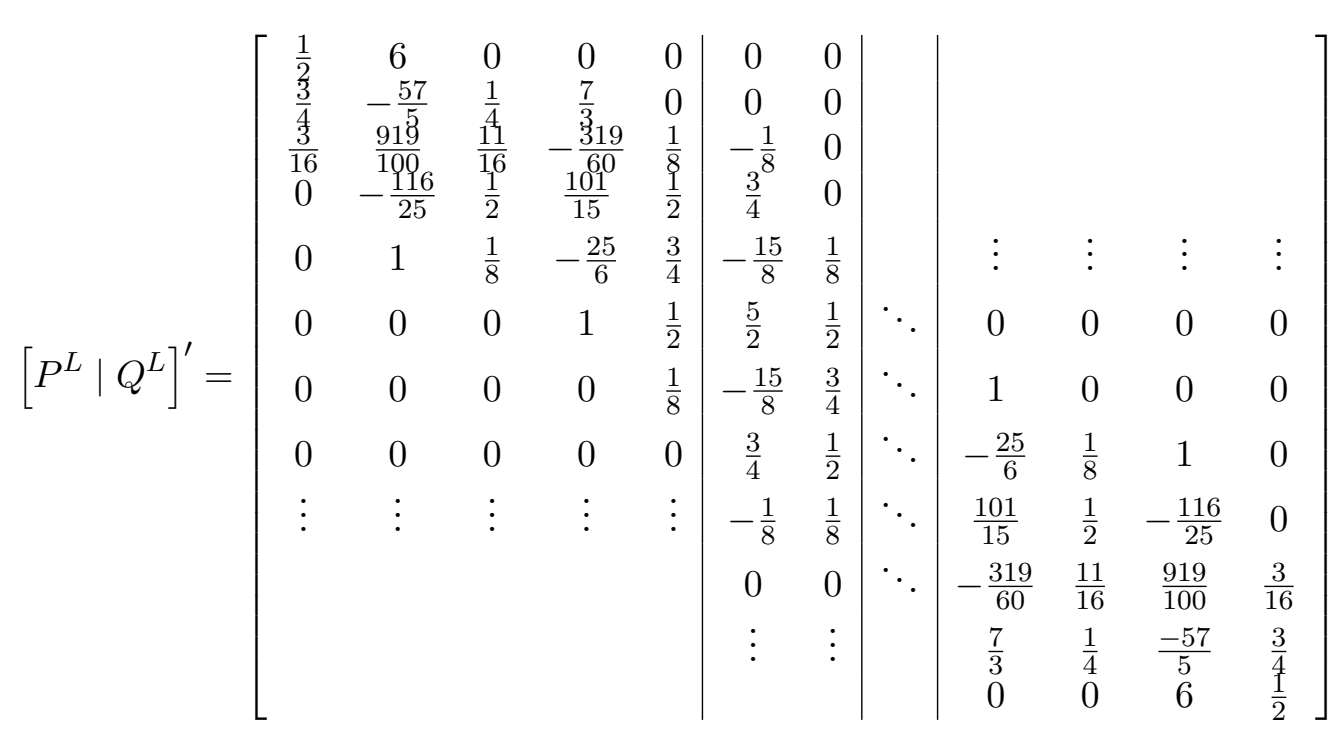

is obtained by permuting the columns of the matrix $\left[P^{L} \mid Q^{L}\right]$ in accordance with the substitution

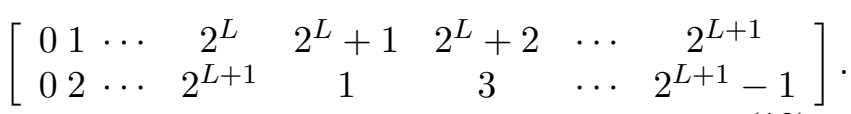

Let the matrix corresponding to the permutation of columns (13) is denoted by $T$. Then the following representation is true [17]

$$
\begin{aligned}
& {\left[P^{L} \mid Q^{L}\right]^{\prime}=\left[P^{L} \mid Q^{L}\right] T .} \\
& {\left[P^{L} \mid Q^{L}\right]_{0-6}^{\prime}=\left[\begin{array}{ccccc|cc|cc}
\frac{1}{2} & 6 & 0 & 0 & 0 & & & \\
\frac{3}{4} & -\frac{57}{5} & \frac{1}{4} & \frac{7}{3} & 0 & 0 & 0 & & \\
\frac{3}{16} & \frac{919}{100} & \frac{11}{16} & -\frac{319}{60} & \frac{1}{8} & -\frac{1}{8} & 0 & & \\
0 & -\frac{116}{25} & \frac{1}{2} & \frac{101}{15} & \frac{1}{2} & \frac{3}{4} & 0 & 0 & 0 \\
0 & 1 & \frac{1}{8} & -\frac{25}{6} & \frac{3}{4} & -\frac{15}{8} & \frac{1}{8} & -\frac{1}{8} & 0 \\
0 & 0 & 0 & 1 & \frac{1}{2} & \frac{5}{2} & \frac{1}{2} & \frac{3}{4} & 0 \\
0 & 0 & 0 & 0 & \frac{1}{8} & -\frac{15}{8} & \frac{3}{4} & -\frac{15}{8} & \frac{1}{8} \\
0 & 0 & 0 & 0 & 0 & \frac{3}{4} & \frac{1}{2} & \frac{5}{2} & \frac{1}{2} \\
& & & & & -\frac{1}{8} & \frac{1}{8} & -\frac{15}{8} & \frac{3}{4}
\end{array}\right],} \\
& {\left[P^{L} \mid Q^{L}\right]_{7-9}^{\prime}=\left[\begin{array}{c|cc|cc}
\frac{1}{8} & -\frac{1}{8} & 0 & & \\
\frac{1}{2} & \frac{3}{4} & 0 & 0 & 0 \\
\frac{3}{4} & -\frac{15}{8} & \frac{1}{8} & -\frac{1}{8} & 0 \\
\frac{1}{2} & \frac{5}{2} & \frac{1}{2} & \frac{3}{4} & 0 \\
\frac{1}{8} & -\frac{15}{8} & \frac{3}{4} & -\frac{15}{8} & \frac{1}{8} \\
0 & \frac{3}{4} & \frac{1}{2} & \frac{5}{2} & \frac{1}{2} \\
& -\frac{1}{8} & \frac{1}{8} & -\frac{15}{8} & \frac{3}{4} \\
& 0 & 0 & \frac{3}{4} & \frac{1}{2} \\
& & & -\frac{1}{8} & \frac{1}{8}
\end{array}\right]}
\end{aligned}
$$

From the representation (14) we find

$$
\left[P^{L} \mid Q^{L}\right]^{-1} \cdot G^{L}=T \cdot\left[P^{L} \mid Q^{L}\right]^{\prime-1} \cdot G^{L} .
$$

Thus, the problem was reduced to finding a solution to the system of matrix equalities

$$
\left[P^{L} \mid Q^{L}\right]_{j}^{\prime}\left(R^{\prime}\right)_{j}^{L}=G_{j}^{L}, j=0,1, \ldots, 2^{L},
$$

which, according to the assumed stepped structure of the matrices $R^{\prime L}$ and $G^{L}$, decomposes into blocks 


$$
\left[P^{L} \mid Q^{L}\right]_{2^{L}-6,2^{L}}^{\prime}=\left[\begin{array}{c|c}
\frac{3}{4} & -\frac{15}{8} \\
\frac{1}{2} & \frac{5}{2} \\
\frac{1}{8} & -\frac{15}{8} \\
0 & \frac{3}{4} \\
& -\frac{1}{8} \\
& \\
&
\end{array}\right.
$$

In this case, the lower right indices in the notation of the left matrices indicate which columns of the matrix $R^{\prime L}$ are calculated by the corresponding system (16), provided that the equations corresponding
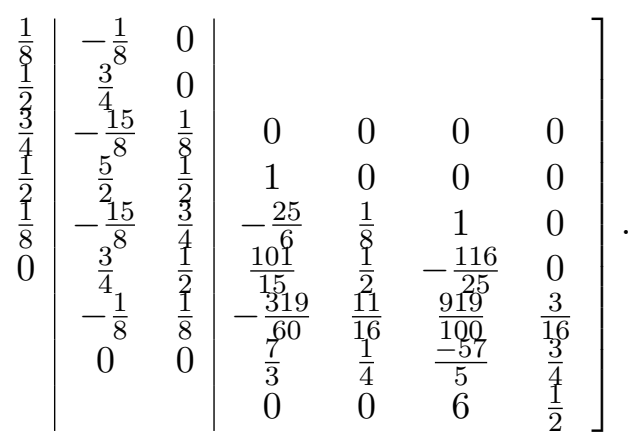

to the zero rows of the matrices $G_{j}^{L}$ removed from the system. As a result, the product of the matrices $\left[P^{L} \mid Q^{L}\right]^{\prime-1} \cdot G^{L}$ turns out to be an almost fivediagonal matrix and has the following form:

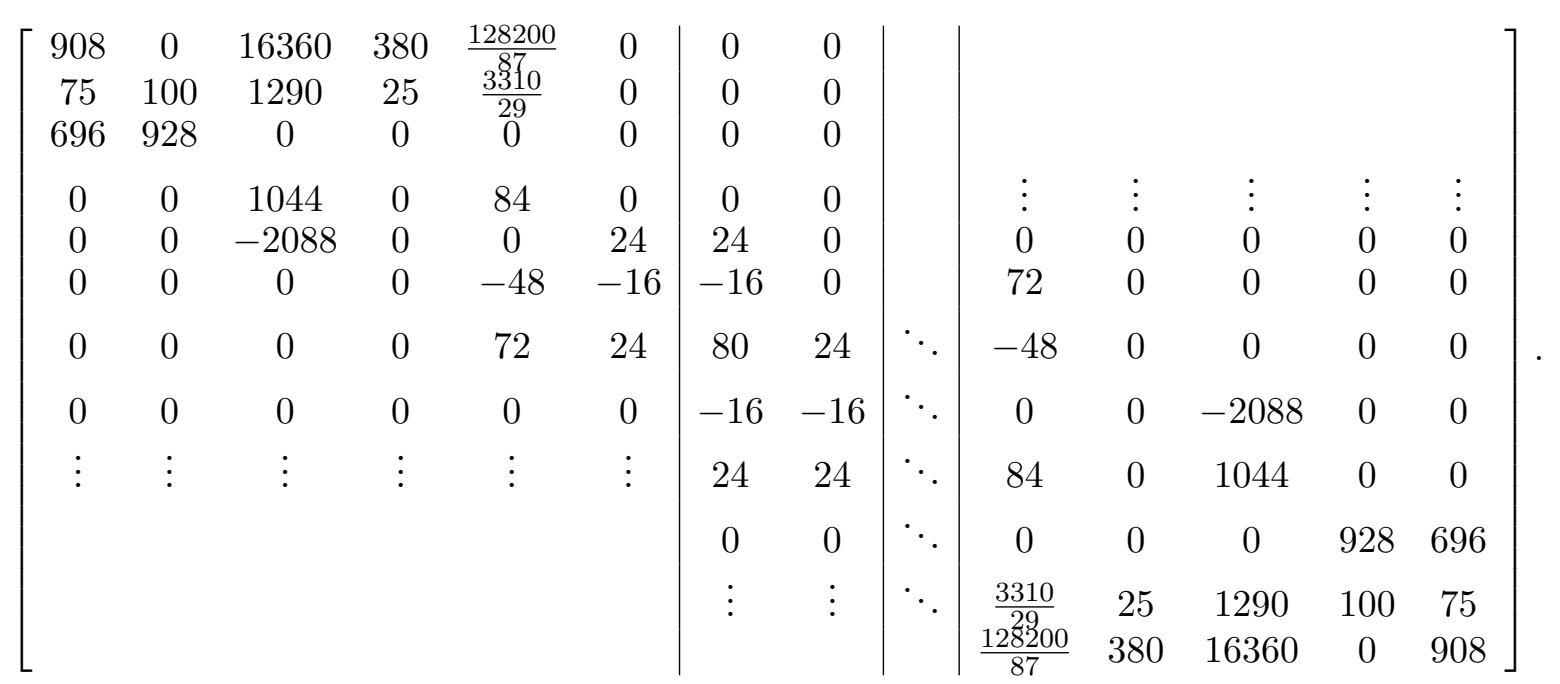

From the equality (15) it follows that to find the matrix $R^{L}=\left[P^{L} \mid Q^{L}\right]^{-1} \cdot G^{L}$, you need to apply to the rows of the matrix (17) the permutation inverse to (13), that is, a permutation in which the records of the pattern and the preimage are swapped. Hence we obtain the required representation of the matrix $R^{L}$.

After that, the solution to the system of equations (9) can be written in matrix form as

$$
\left[\frac{C^{L-1}}{D^{L-1}}\right]=\left[P^{L} \mid Q^{L}\right]^{-1} C^{L}=R^{L}\left(G^{L}\right)^{-1} C^{L} .
$$

So, instead of directly solving a system of the form (9), we can solve the system

$$
G^{L} \Xi^{L}=C^{L}
$$

with respect to some values of $\Xi^{L}$ and then just calculate the values of $C^{L-1}$ and $D^{L-1}$ using the linear transformation $\left[\frac{C^{L-1}}{D^{L-1}}\right]=R^{L} \Xi^{L}$.
Thus, in contrast to [11], in the five-diagonal approach, there is no possibility to perform transformations with the boundary rows of the splitting matrix (with the corresponding boundary wavelets) to ensure a strict diagonal predomination and consequently the stability of the calculations.

Nevertheless, for large values of $L$, we still need to divide the system (18) into even and odd numbers to reduce the algorithm to solving a five-diagonal system of equations. We can achieve this goal by combining the five-diagonal sweep method with the incomplete reduction method studied in $[18, \mathrm{Ch}$. IV, $\S 3]$.

In a real situation of wavelet analysis of a discrete signal, the homogeneous boundary conditions necessary for the creation of a wavelet decomposition are not satisfied. Therefore, before applying the above algorithm, it is necessary to subtract values of the line $f(a)+(f(b)-f(a))(x-a) /(b-a)$ from the set values. After wavelet analysis of obtained differences and reconstruction on wavelet coefficients of the approximating spline of the third degree, values of this line are added to it. 


\section{Modeling of road pavements based laser scanned data}

Laser scanning is a new direction in high-precision 3D-measurements [19]. As to mobile scanning, scopes of application include positioning of the automobile and railroads, bridges, overpasses, city streets, the coastline, etc. The main advantage of laser scanning is the possibility to work at objects with heavy traffic, at industrial facilities without stopping of production and in hard-to-reach sites, and also at objects having a complex configuration [20]. Cartesian components of the GPS data from laser scanning device are issued in the form of the array ("cloud") of points, in which there is no division into separate cross scans. As the principle of mobile scanning system allows working on the road without traffic overlapping, the cloud of points contain a roadside landscape and hindrances on the carriageway (reflection from people who are on an object, technique, vegetation, etc.) (Fig.1).

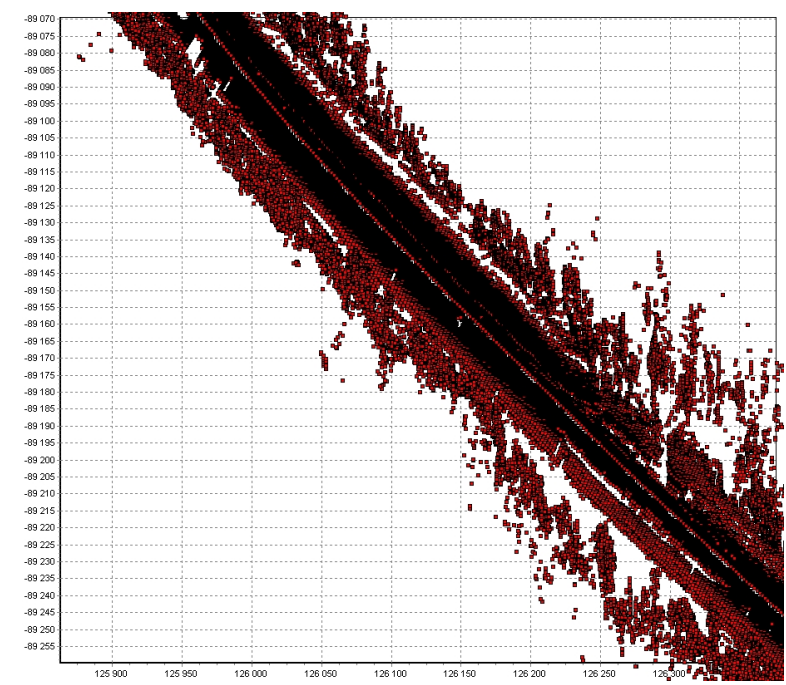

Figure 1: Isometric projection of cloud points for a small section of the highway

Main goals of preliminary processing of laser scanned data are the removal of a roadside and surrounding landscape, the filling of gaps in the scanned data, created by cars passing by on the carriageway, and the elaboration of the planned axial line of the road. From the mathematical point of view the production of the axis of the road allows transforming bends of the highway to some rectangular area, for which it is possible to apply standard methods of twodimensional spline interpolation [21] on a rectangular grid, keeping structural lines of the road (edges, brows), unlike the popular method of restoration of a surface of the highway by triangulation of chaotic points [22]. Very important is that at such approach high precision of detection of cracks and damages of a road pavement in the places demanding repair is guaranteed and construction and application of the wavelet transformation of interpolation splines for compression of the scanned information in the places of highways not demanding repair is significantly facilitated also. Figure 2 shows the results of the data wavelet compression for a single track. In this case, a very small section of the track is shown. The total length of the track exceeds $4 \mathrm{~km}$. Compression with a coefficient of 15.9 is achieved. The maximum resulting error does not exceed $3.5 \mathrm{~cm}$.

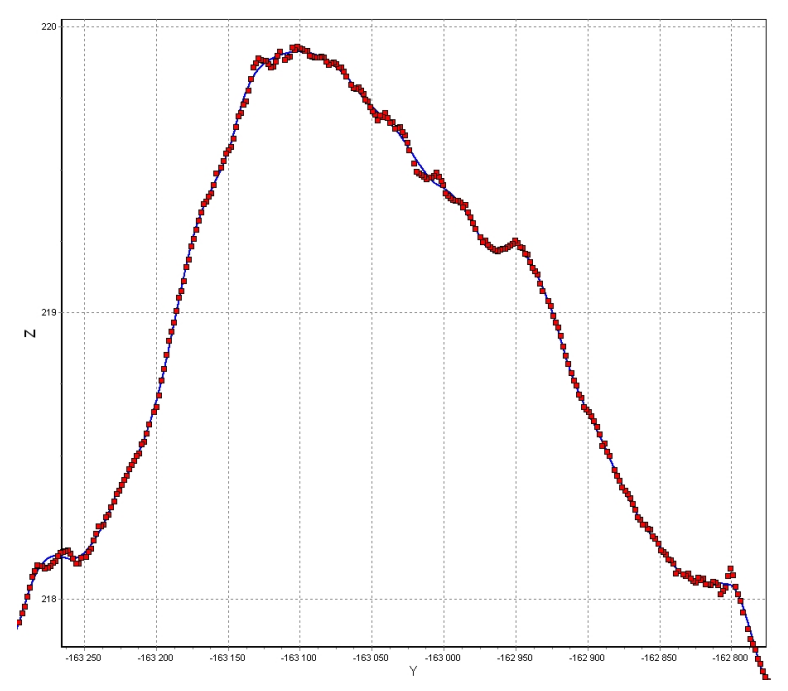

Figure 2: Data compression results for a single track. The total length of the track is more than $4 \mathrm{~km}$. The Y-Z profile of the section with a length of $450 \mathrm{~m}$ is shown

Note that when using the wavelet transformations based on Hermite splines it is necessary to calculate approximate values of derivatives in nodes of the densest grid with suitable accuracy (for example via ENO-scheme [23]) to apply algorithms of wavelet transformations. From the point of view of compression the number of wavelet-coefficients given thus in comparison with the methods, based on B-splinewavelet-transformations, will multiply increase. The stated above algorithms were the basis for the software package for the processing of laser scanned data. Results of visualization of the data processed are given in Fig.3, 4.

When compressing data using bicubic wavelets with a compression ratio of 3,97 , the resulting rootmean-square error of the surface approximation does not exceed $5.7 \mathrm{~mm}$. The mathematical expectation of the deviation is slightly biased - by $9.4 \cdot 10^{-4} \mathrm{~m}$. A three-dimensional image of a part of the surface after wavelet compression is shown in Fig.4. 


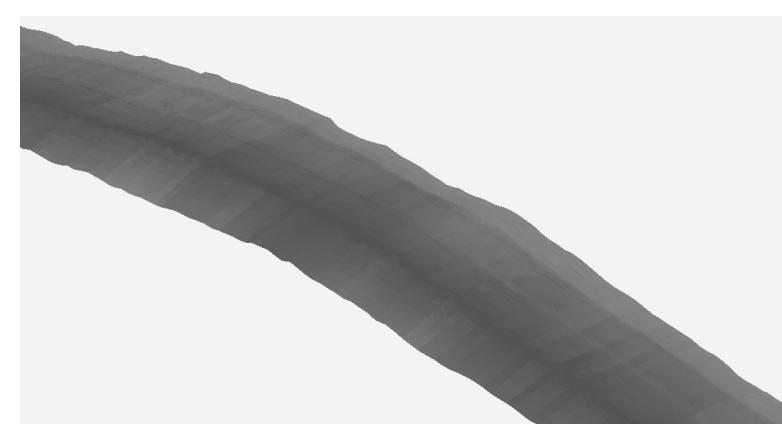

Figure 3: A piece of the surface to compress the bicubic wavelets

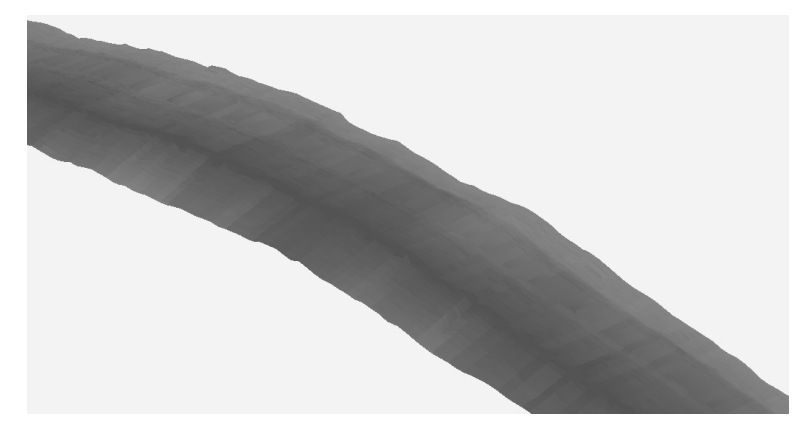

Figure 4: A piece of the surface after compression bicubic wavelets

\section{Conclusion}

The article discusses the further development of the author's procedure for an even-odd partition of the defining system of the Hermite wavelet expansion for the practically important case of approximating functions that do not require specifying the values of the derivatives, based on $B$-splines of the third degree. In this paper, we propose using an algorithm based on wavelet transform to detect cracks and damages of a road pavement. Besides, we design a filter needed in the transform and make the computation much more efficient by block matrix identities. The figures and data resulting from experiments show that a bicubic wavelet transform is a powerful tool in cracks and damages of road pavement detection.

The extension of the proposed approach to splines of a higher degree and a larger number of zero moments can provide new opportunities for the development of algorithms for constructing and applying spline wavelets. We believe that the flexible multiscale decomposition of the wavelet transform has the potential to be used more and more in image and video processing. And we expect a combination of cracks and damages of road pavement detection and machine learning in the future to develop a more intelligent road repairing system.
References:

[1] R. Wilson, Multiresolution image modeling, Electronics and Communications Engineering Journal, Vol.9, No.2, 1997, pp. 90-96.

[2] I. Daubechies, Ten lectures on wavelets, Society for Industrial and Applied Mathematics, Philadelphia (PA), 1992.

[3] S. Mallat, A Wavelet Tour of Signal Processing, Academic Press, San Diego (CA), 1999.

[4] E.J. Stollnitz, T.D. DeRose, D.H. Salesin, Wavelets for Computer Graphics, Morgan Kaufmann Publishers, San Francisco, 1996.

[5] C.K. Chui, An Introduction to Wavelets, Academic Press, New York, London, 1992.

[6] V. Strela, Multiwavelets: Theory and Applications, PHD Thesis (Math.), Cambridge, Massachusetts, 1996.

[7] W. Dahmen, B. Han, R.Q. Jia et al., Biorthogonal multiwavelets on the interval: cubic Hermite splines, Constructive Approximation, Vol.16, 2000, pp. 221-259.

[8] R. Warming, R. Beam, Discrete multiresolution analysis using Hermite interpolation: biorthogonal multiwavelets, SIAM Journal on Scientific Computing, Vol.22, No.1, 2000, pp. 269-317.

[9] B.M. Shumilov, A.N. Baigulov, A study on modeling of road pavements based on laser scanned data and a novel type of approximating hermite wavelets, WSEAS Transactions on Signal Processing, Vol.11, 2015, pp. 150-156.

[10] M.W. Frazier, An Introduction to Wavelets through Linear Algebra, Springer-Verlag, New York, 1999.

[11] B.M. Shumilov, Shifted cubic spline wavelets with two vanishing moments on the interval and a splitting algorithm, WSEAS Transactions on Systems, Vol.19, 2020, pp. 149-158.

[12] C. De Boor, A Practical Guide to Splines, Applied Mathematical Sciences, Vol.27, SpringerVerlag, New York, 1978.

[13] K. Koro, K. Abe, Non-orthogonal spline wavelets for boundary element analysis, Engineering Analysis with Boundary Elements, Vol.25, 2001, pp. 149-164.

[14] D. Cerná, Cubic spline wavelets with four vanishing moments on the interval and their applications to option pricing under Kou mode, 
International Journal of Wavelets, Multiresolution and Information Processing, Vol.17, No.1, 2019, article No. 1850061.

[15] B.M. Shumilov, S.M. Matanov, Supercompact cubic multiwavelets and algorithm with splitting, 2011 International Conference on Multimedia Technology, 2011, pp. 2636-2639.

[16] B.M. Shumilov, Cubic multiwavelets orthogonal to polynomials and a splitting algorithm, $\mathrm{Nu}$ merical Analysis and Applications, Vol.6, No.3, 2013, pp. 247-259.

[17] S. Pissanetzky, Sparse Matrix Technology, Academic Press, London, 1984.

[18] A.A. Samarskii, E.S. Nikolaev, Numerical Methods for Grid Equations, Vol. I Direct Methods, Birkhauser, Basel, 1989.

[19] W. Boehler, A. Marbs, 3D Scanning Instruments, Proc. of the CIPA WG6 Int. 2002. Workshop on scanning for cultural heritage recording. http://www.isprs.org/commission5/workshop/

[20] H.C. Yun, M.G. Kim, J.S. Lee, Applicability estimation of mobile mapping system for road management, Contemporary Engineering Sciences, Vol.7, 2014, pp. 1407-1414.
[21] G. Zhao, S. Xu, W. Li, X. Zhu, Wavelets-based multiresolution representation and manipulation of closed $B$-spline curves, Proceedings of $I C$ SEC, 2002, pp. 490-493.

[22] J.D. Boissonnat, O. Devillers, S. Pion, M. Teillaud, M. Yvinec, Triangulations in CGAL, Computational Geometry, Vol.22, 2002, pp. 5-19.

[23] F. Aràndiga, A. Baeza, R. Donat, Discrete multiresolution based on hermite interpolation: computing derivatives, Communications in Nonlinear Science and Numerical Simulation, Vol.9, 2004, pp. 263-273.

\section{Creative Commons Attribution License 4.0 (Attribution 4.0 International, CC BY 4.0)}

This article is published under the terms of the Creative Commons Attribution License 4.0

https://creativecommons.org/licenses/by/4.0/deed.en_US 